\title{
Designing escape routes for buildings through an aggregate approach
}

\author{
M. Di Gangi \\ Department of Civil and Environmental Engineering, Informatics and \\ Applied Mathematics, University of Messina, Italy
}

\begin{abstract}
In this paper a method for the definition of effective destination and escape routes by means of the simulation of pedestrian outflow related to the evacuation of a building is described. The generated feasible alternatives are compared in terms of evacuation time by means of an aggregate assignment model. Such a method can be easily implemented in a spreadsheet and can be used to give a first evaluation of evacuation procedures without performing evacuation drills. It can also be used to give a fast response in identifying critical points on the network. To check the capabilities of the proposed procedure, results obtained from simulations are compared with data recorded from experimentation on a test site conducted in a primary school located in an Italian town.
\end{abstract}

Keywords: evacuation, simulation.

\section{Introduction}

One of the aspects that in the last years attracted the attention of some experts of the field on transport model consists in the analysis of working modality of a transport system when it is subjected to emergency conditions. Such approach should allow the availability of tools able to conduct simulations of evacuations avoiding expensive drills or to give useful indications to enhance evacuation processes of different extended areas (building, industrial site, town, etc.).

An evacuation can be defined as a general mobilization of people (and/or goods) due to the occurrence of a calamitous event. Its main objective is to reduce the number of people (and/or goods) present in the area where the event strikes [1]. Evacuation drills are mainly performed to practice the people to leave the interested area; these tests can also be used for getting information 
concerning the behavior of the people in order to build a set of mathematical models able to reproduce the evacuation. These models can constitute a Decision Support System (DSS) to be used for planning emergences [2].

Pedestrian evacuation has been considered in the last years a lead issue from both theoretical and practical point of view. On the one hand details of pedestrian evacuation have been cast into mathematical formulations yielding to various representations of pedestrian interactions. On the other hand theoretical developments have conducted to model emergency evacuation and situations [312] using sophisticated simulation methodologies.

Computational complexity of proposed models requires the analysis outflow conditions of networks, and the adoption of dedicated and sophisticated tools. On the other hand there is also, in certain circumstances, a need for simple tools able to quantify, in terms of evacuation time, the effectiveness of an evacuation plan.

Such aggregate approaches, in order to simulate pedestrian movements, consider laws of motion of pedestrian flow using, in general, relationships between speed and density [13-16] or deriving from continuum theory of traffic flows $[17,18]$.

In this paper a specific methodology (models and procedure) to define effective escape routes by simulating evacuation of buildings is shown. The proposed aggregate approach allows a prompt first attempt evaluation in case of the lack of commercial software or tools suitable to perform advanced and more sophisticated simulations.

The paper is structured as follows: in section 2 it is described the proposed method to define evacuation routes in case of buildings, in section 3 results of an application to a real case represented by a provisional school unit built after L'Aquila earthquake is presented and some remarks are reported in section 4.

\section{Methodology}

\subsection{Definition of evacuation scenarios}

Steps followed to define evacuation scenarios are described in the following and their connections are sketched in Fig. 1.

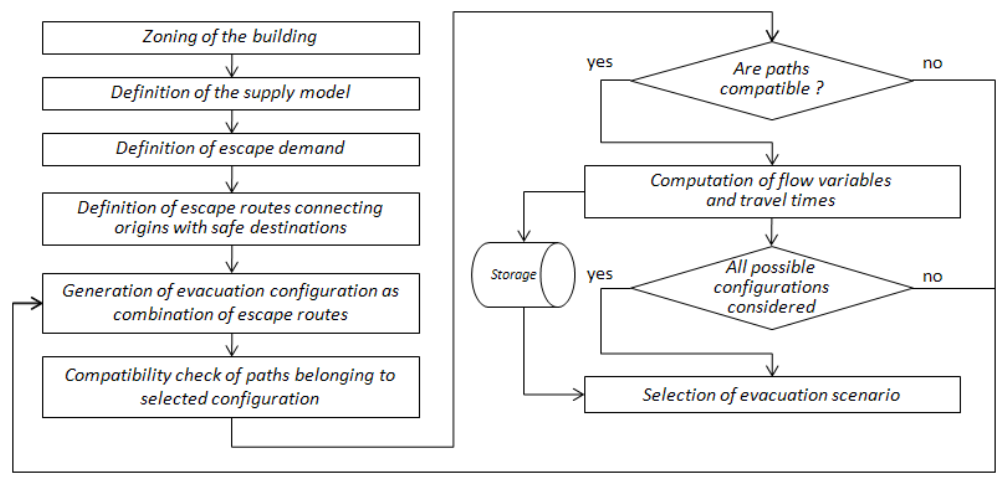

Figure 1: Scheme of the activities to define evacuation scenarios. 


\subsubsection{Zoning of the building}

Every starting and terminal area is identified within the building (and eventually its surroundings if safe areas are located outside the building) yielding to the definition of origin and destination zones. Every zone is approximated with a single point (zone centroid) representing origin or destination nodes of escape routes, that can be divided in:

- zone centroids: these nodes represent the barycentre of each zone (rooms, offices, etc.) comprising the building. They sum up the origins of the trips of all the people who, at emergency time, are within the considered area making up a zone. There is a centroid for each zone in which the building is divided;

- destination nodes (centroids): these represent the safe areas towards which people converge in an emergency; they correspond to the destinations of evacuation routes;

\subsubsection{Definition of the supply model}

In this step geometric characteristics of elements of the building are acquired; then, considering the elements of a building, the classes of components making up the graphs are:

- network nodes: these are located at each potential change of direction along a generic evacuation route or at significant variations in geometric and/or functional characteristics of a trunk (i.e. width variations);

- real arcs: these represent the connection between two network nodes or a network and a destination node; they coincide with trunks of the pedestrian network and are classified into flat ramps (corridors) and descending or ascending ramps (stairs);

- connector arcs: these are used to represent the connection between an area centroid and a network node.

\subsubsection{Definition of escape demand}

In this step data concerning occupants, defining both their number and their positions, are acquired. Those values are attributed to the correspondent origin nodes representing each considered site (zone centroid). Different scenarios should be considered for the analysis of evacuation considering different numbers and distributions of occupants.

\subsubsection{Generation of escape routes: path generation}

After building the graph of the network representing the elements, in term of components of the escape ways (ramps, corridors, stairs, etc.) of the building, a set of evacuation routes connecting each origin with each defined safe destination is generated. In order to explore a wide range of alternatives, for each origin-destination pair more than one path is taken into consideration. To define these evacuation routes a set of paths is generated considering a $\mathrm{K}$ shortest loopless path algorithm considering as arc cost its travel time. One of the possibilities, in order to take into account of the environmental condition of each link within conventional path search algorithms, can be to weight travel time associated to the generic arc of the graph by means of the level of risk associated to the arc [19], assuming that the role of risk can be associated similarly to the 
one played by saturation level in congested networks. The relationship between the weighted arc cost and the travel time can be written as follows:

$$
T w_{i}=T t_{i} \cdot\left\{1+\alpha\left[\ln \left(1 / s_{i}\right)\right]^{\beta}\right\}
$$

where:

$T w_{i} \quad$ is the weighted cost associated to arc $\mathrm{i}$;

$T t_{i} \quad$ is the travel time of arc $\mathrm{i}$;

$s_{i} \quad$ is a safety probability $0<s_{i} \leq 1$;

$\alpha, \beta \quad$ are parameters.

The level of the reliability of an arc (safety probability) depends on the nature of the considered event (i.e. an evacuation route using downstairs can be considered safe in case of fire but can be unsafe in case of flood).

\subsubsection{Generation of feasible alternatives}

The number of alternative is strictly influenced by the value assumed the number $\mathrm{K}$ of alternative evacuation routes considered for each origin/destination pair, since the number of potential alternatives is given by the permutation of the $\mathrm{K}$ paths on $\mathrm{N}_{\text {od }}$ origin/destination pairs, that is $\mathrm{N}_{\mathrm{od}}{ }^{\mathrm{K}}$ (in the hypothesis that for every pair it is possible to identify k different paths; in general if $\mathrm{K}_{\mathrm{i}}$ is the number of path for the $i$-th pair, the number of potential alternatives is given by $\Pi_{i \in \text { od }} K_{i}$ ).

The scenario is generated by applying a selection rule to the set of potential alternatives. The rule here introduced, in order to define a feasible set, consists in eliminating those combinations where contra flow is potentially allowed; this implies that each element of the network (ramp, corridor, stair, etc) can be covered in one way only.

In order to establish feasibility, given a path $\mathrm{k}$ let $\mathrm{l}_{\mathrm{k}}(\mathrm{r}, \mathrm{s})$ be a generic arc belonging to path $\mathrm{k}$ connecting nodes $\mathrm{r}$ and $\mathrm{s}$; two paths, $\mathrm{h}$ and $\mathrm{k}$, are compatible if, considering each arc $\mathrm{l}_{\mathrm{h}}(\mathrm{r}, \mathrm{s})$ of path $\mathrm{h}$, it does not exist an $\operatorname{arc} \mathrm{l}_{\mathrm{k}}(\mathrm{s}, \mathrm{r})$ belonging to path $\mathrm{k}$. Such rule is implemented by defining a compatibility incidence matrix $\boldsymbol{C}\left[\mathrm{n}_{\mathrm{k}} \mathrm{X} \mathrm{n}_{\mathrm{k}}\right.$ ] where the generic element $c_{h k}$ is equal to 1 if paths $\mathrm{h}$ and $\mathrm{k}$ are compatible, otherwise it is equal to 0 .

\subsection{Computing flow variables and evacuation time}

The proposal of this method arises from the need to have a tool, even though approximate, able to give some indications both on the outflow conditions and on the evacuation times of a building. One of the advantages of this method consists on the possibility to evaluate evacuation times with the only support of a common worksheet application, avoiding the necessity to use specific commercial software.

\subsubsection{Main hypotheses}

The proposed method of estimating evacuation time is based on the following assumptions:

- all occupants will begin evacuation at the same time and will not hinder each other;

- occupants will evacuate via a previously defined escape route; 
- initial walking speed depends on the density of persons, assuming that the flow is only in the direction of the escape route, and that there is no overtaking;

- full availability of escape arrangements is considered, unless otherwise stated;

- people can move unhindered;

- effects of passenger age and mobility impairment, flexibility of arrangements, unavailability of corridors, restricted visibility due to smoke, can be accounted for in specific correction factors.

Proposed method can be summarized through a succession of operations finalized to the evaluation of evacuation time.

\subsubsection{Demand-supply interaction model}

- Computation of occupants for each element $q_{i}$. For each element of escape routes, the flow of occupants (vector $\boldsymbol{q}$ ) is computed using the arc-path incidence matrix ( $\boldsymbol{A})$ as $\boldsymbol{q}=\boldsymbol{A d}$, where $\boldsymbol{d}$ is the vector of demand.

- Computation of the specific flow $\left(q_{s}\right)$. This value is computed dividing flow $q$ by the effective width $w$ of the considered element except for connectors; for those latter components specific relationships based on density are adopted.

- Computation of speed. Once specific flow $q_{s}$ is known, the following two cases arise:

- values of $q_{s}$ do not reach the value $q_{s}^{\max }$ of characteristic maximum specific flow for the considered element; speed $v$ ' is computed using specific relationships depending on specific flow;

- values of $q_{s}$ overtake the value $q_{s}^{\max }$ of characteristic maximum specific flow for the considered element; in this case queues arise in correspondence of transition points; correspondent speed $v$ ' is given by the limit value indicated by relationships expressing speeds depending on specific flow.

\subsubsection{Computation of times}

- Computation of flow time for each element $\left(t f_{i}\right)$. Those values are computed once evaluated walking speed for each considered element as $t t_{i}=l_{i} / v_{i}$.

- Computation of queue time for each element $\left(t q_{i}\right)$. Those values are computed depending on specific flow $q_{s}$ and the value $q_{s}{ }^{\max }$ of characteristic maximum specific flow for the considered element as $t q_{i}=\left(q_{s}-q_{s}^{\max }\right) / q_{s}^{\max }$.

- Computation of travel time for each escape route (T). Travel times are computed, for the whole set of evacuation routes, considering the arc-path incidence matrix as $\boldsymbol{T}=\boldsymbol{A}^{T}\left(\boldsymbol{t}_{\boldsymbol{f}}+\boldsymbol{t}_{q}\right)$.

- Computation of evacuation time. After computing travel time for each evacuation route, the higher value $T$ among all the travel times is considered as the evacuation time. 


\section{Applicative context}

\subsection{Model calibration}

The part of the methodology concerning demand/supply interaction has been calibrated in a real context [20-24] where evacuation drills have been carried out in order to collect data to validate proposed models. In particular two evacuation tests (drills) have been carried out and data were gathered concerning supply and demand. During the drills a monitoring system was arranged, with manual/automatic tools and 12 video cameras, in order to acquire data concerning pedestrian outflow (times, densities) both inside and outside the building until the gathering places were reached.

\subsection{Application of the methodology}

The application of the methodology was performed to define the evacuation routes on one of the provisional school units (MUSP - Lotto 16) built after L'Aquila earthquake of April $6^{\text {th }}$ 2009. Plans of the two floors of the considered building are sketched in Fig. 1. In the following of this paragraph operations conducted at each step of the proposed procedure in the considered context are explained.

\subsubsection{Zoning of the building}

Considering each room as a distinct zone, 39 potential origins and 2 destinations outside the building have been identified. For the sake of simplicity dressing rooms have not been considered as origin. Origins are treated as actual if, in the considered scenario, the presence of people attributed to the area they represent is thought. A centroid has been attributed to each zone.

\subsubsection{Definition of the supply model}

The building has been schematized considering the network of all possible pathways. Corridors were considered in both directions and a specific arc has been considered for each door aperture. The resulting plan, shown in Fig. 2, consists of 170 nodes and 189 arcs (note that arcs related to vertical connections - stairs - are not visible).

\subsubsection{Definition of escape demand}

In the considered scenario the hypothesis made is that only classrooms and offices are in use, no activities are conducted in laboratories and in the school gym and there is no one in the toilets. So, only 25 of the potential 39 origins are then considered, and occupants of each room correspondent to the origin node have been defined as indicated in Tab. 1.

Following these hypotheses, the total number of occupants to evacuate is of 451 people, 330 located at the first floor and 121 located at the ground floor.

\subsubsection{Definition of escape routes}

Paths connecting the 25 origins with the 2 assembly point have been generated. In order to explore a wide range of alternatives, for each origin-destination up to 


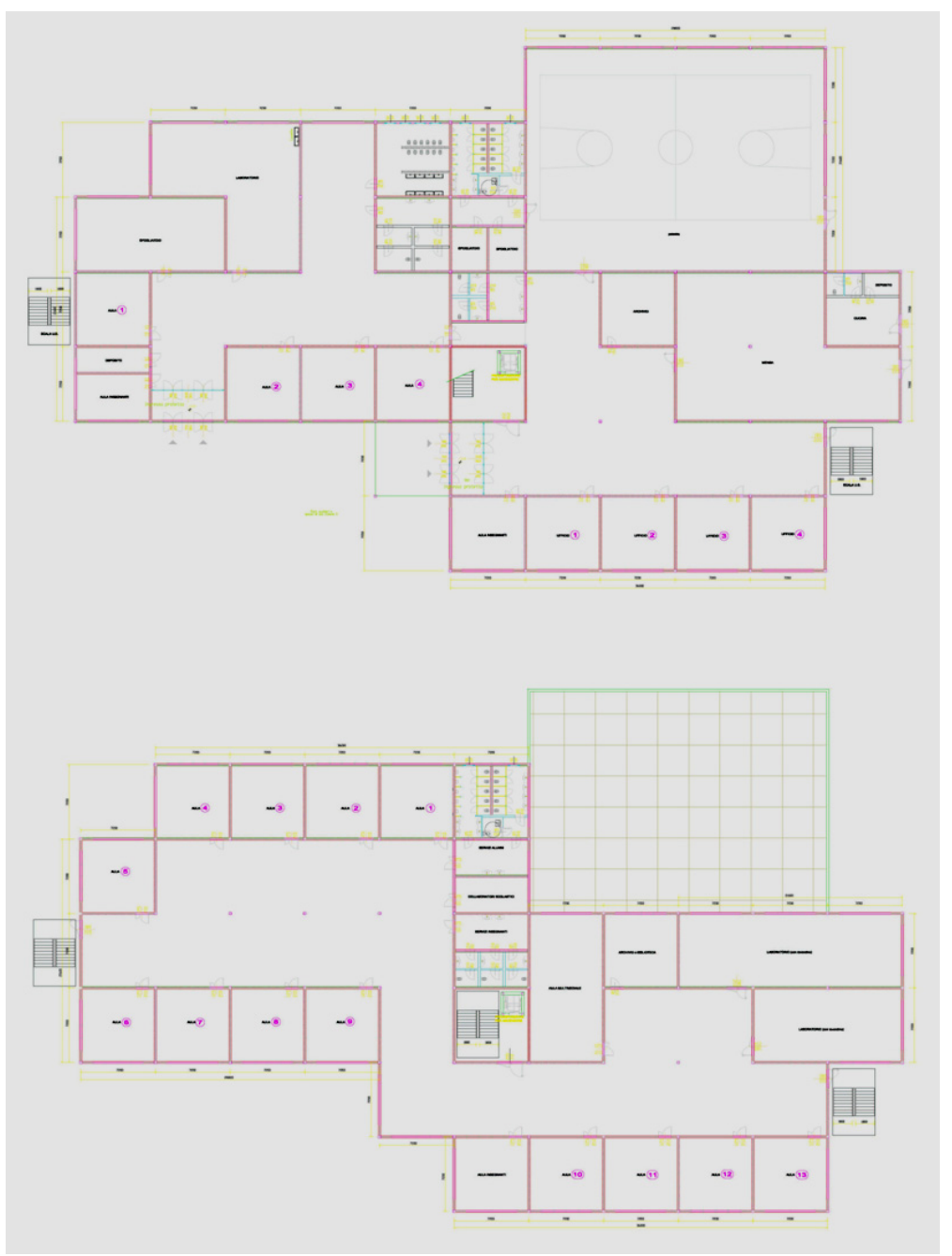

Figure 2: $\quad$ Plan of the ground and first floors of the provisional school units.

Table 1: $\quad$ Definition of occupants for each origin node.

\begin{tabular}{|c|c|c|c|c|c|c|c|c|c|c|c|c|c|c|}
\hline \multicolumn{15}{|c|}{ Ground floor } \\
\hline Origin node & 1 & 3 & 4 & 5 & 6 & 11 & 12 & 13 & 14 & 15 & 16 & & & \\
\hline Occupants & 25 & 5 & 25 & 25 & 25 & 5 & 2 & 2 & 2 & 2 & 3 & & & \\
\hline \multicolumn{15}{|c|}{ First floor } \\
\hline Origin node & 85 & 86 & 87 & 88 & 89 & 90 & 91 & 92 & 93 & 94 & 95 & 96 & 97 & 98 \\
\hline Occupants & 25 & 25 & 25 & 25 & 25 & 25 & 25 & 25 & 25 & 5 & 25 & 25 & 25 & 25 \\
\hline
\end{tabular}


3 evacuation routes for each O/D pair have been taken into consideration. Freeflow time has been considered as cost of each arc; it is given by arc length divided by its free flow speed, supposed equal to $0.8 \mathrm{~m} / \mathrm{s}$ for corridors and $0.5 \mathrm{~m} / \mathrm{s}$ for descending stairs. A k-shortest path algorithm has been used to evaluate escape routes; results consist of 59 generated evacuation routes since, for some $\mathrm{O} / \mathrm{D}$ pair, only 1 or 2 path are obtainable.

\subsubsection{Generation of evacuation configurations}

Considering the set of evacuation routes computed above, feasible evacuation scenarios have been built taking account of the selection rule consisting in eliminating those combinations where contra flow is potentially allowed. So, starting from the 306,110,016 possible combinations obtained combining paths for all the O/D pair each other, the application of such selection rule reduces the number of feasible configurations to 22,712 .

\subsubsection{Computation of flow variables and travel times and selection of scenario}

For each one of the feasible configurations, using the aggregate model described before, travel times and flow characteristics are computed in order to define indicators on evacuation conditions. The effective scenario has been identified considering, among all the simulated configurations, the one that minimize evacuation time. The selection of a configuration implies the attribution of the safe area to be reached from evacuees; attribution of destinations (safe area) to each origin (room) is shown in Tab. 2. In Tab. 3 some statistics expressed in terms of evacuation time and total time are shown, whilst indicators obtained for the selected configuration are summarized in Tab. 4.

Table 2: $\quad$ Definition of safe area for each origin node.

\begin{tabular}{l|rrrrrrrrrrrrrrr}
\hline \multicolumn{11}{c}{ Ground floor } \\
\hline Origin node & 1 & 3 & 4 & 5 & 6 & 11 & 12 & 13 & 14 & 15 & 16 & & \\
Destination (Safe area) & 78 & 78 & 78 & 78 & 78 & 78 & 78 & 78 & 78 & 78 & 78 & & \\
\hline \multicolumn{110}{c}{ First floor } & & & & & & & & \\
\hline Origin node & 85 & 86 & 87 & 88 & 89 & 90 & 91 & 92 & 93 & 94 & 95 & 96 & 97 & 98 \\
Destination (Safe area) & 78 & 78 & 78 & 78 & 78 & 78 & 78 & 78 & 170 & 78 & 170 & 170 & 170 & 170 \\
\hline
\end{tabular}

Table 3: $\quad$ Statistics obtained for the whole set of configurations.

\begin{tabular}{lcccccc}
\hline & \multicolumn{2}{c}{ Min } & \multicolumn{2}{c}{ Max } & & \\
& Time [s] & Scenario & Time [s] & Scenario & Mean [s] & Var. [s ${ }^{2}$ ] \\
\hline Evacuation time & 445 & 90 & 1432 & 232 & 744 & 9275 \\
Total time & 153,024 & 19,963 & 480,711 & 232 & 216,296 & $7.77 \mathrm{E}+08$ \\
\hline
\end{tabular}


Table 4: $\quad$ Indicators obtained for optimal configuration.

\begin{tabular}{lrr}
\hline \multicolumn{1}{c}{ Indicator } & Time [s] & Time [hh:mm:ss] \\
\hline Evacuation time & 445 & $00: 07: 25$ \\
Total time & 154,327 & $42: 52: 07$ \\
Max queue time & 279 & $00: 04: 39$ \\
\hline Total queue time & 93,097 & $25: 51: 37$ \\
\hline
\end{tabular}

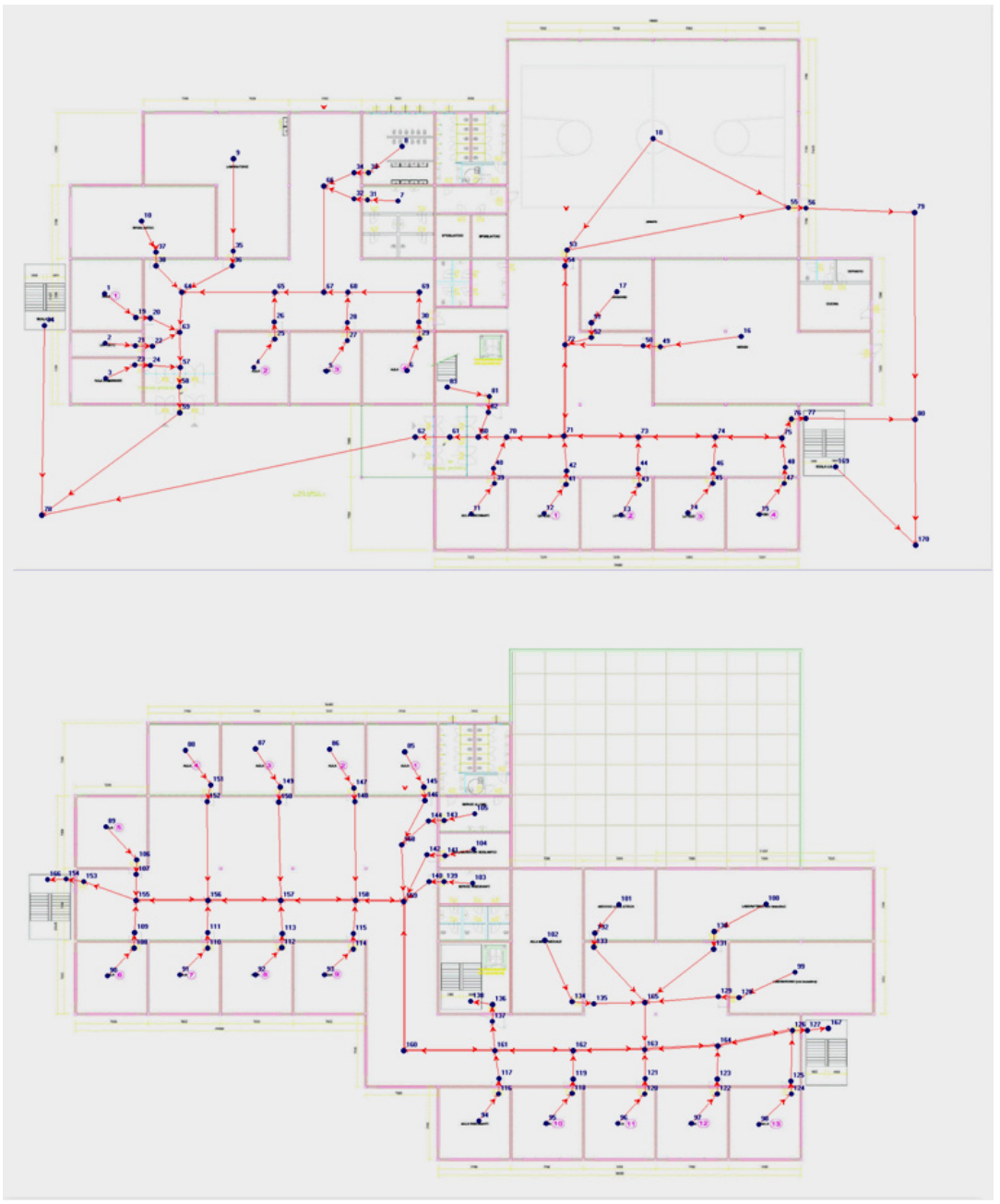

Figure 3: Plans of the pedestrian network of the provisional school unit. 


\subsection{Computational issues}

Since one of the aim of this work was to build up a simple tools able to quantify, in terms of evacuation time, the effectiveness of an evacuation plan, the whole procedure, except for the zoning and the graph definition, has been implemented in a Microsoft Excel ${ }^{\odot}$ spreadsheet where the modules of the procedure were coded using the VBA language.

Running times for each step, obtained on an AMD Athlon II, 2 GB RAM with Windows 7 and Office 2007, are shown in Tab. 5.

Table 5: $\quad$ Running time for each step of the procedure.

\begin{tabular}{lr}
\hline \multicolumn{1}{c}{ Step } & Time [s] \\
\hline Path generation & 0.406 \\
Evaluation of path compatibility & 3,543 \\
Generation of alternatives & 1,328 \\
Evaluation of alternatives & 40,387 \\
\hline
\end{tabular}

\section{Conclusions and perspectives}

The main result of this paper concerns a method for the definition of effective escape routes by means of the simulation of pedestrian outflow related to the evacuation of a building using an aggregate model for the estimation of evacuation time. A comparison between experimental data and simulation results shows how the usage of appropriate simulation models can realistically reproduce user behavior. It was shown that such models could be used as a support both to verify effectiveness of existing evacuation plans without resorting to expensive drills and to draw up evacuation plans and can be used to give a fast response in identifying critical points on the network. The capabilities of the proposed approach have been shown by means of an application on a real case.

Implementation of appropriate cost functions can make the applied methodologies suitable for any building and/or area with homogeneous characteristics in terms of activities. Further investigations on travel time functions under different operative conditions are under development.

\section{Acknowledgements}

The author wishes to thank Prof Mauro Dolce and Dr Elvezio Galanti, members of the Italian Civil Protection, for their contribution in the availability of details on the provisional school unit of L'Aquila considered in the application. 


\section{References}

[1] Russo F., Chilà G., A sequential dynamic choice model to simulate demand in evacuation conditions. Proc. of Risk analysis VII \& Brownfields V, Brebbia C.A. (ed.), WIT Press, Southampton, 2010, pp. 431-442.

[2] Russo F., Rindone C., Evaluation methods for evacuation planning. Proc. of Urban Transport XVI, Urban Transport and the Environment in the 21st century, Brebbia C.A. (ed.), WIT Press, Southampton, 2010, pp. 335-343.

[3] Helbing, D., Farkas, I.J., Molnár, P., Vicsek, T., Simulation of Pedestrian Crowds in Normal and Evacuation Situations. M. Schreckenberg and S. D. Sharma (eds.) Pedestrian and Evacuation Dynamics, Springer, 2002, pp. 21-58.

[4] ASERI (Advance Simulation of Evacuation of Real Individuals) A model to simulate evacuation and egress movement based on individual behavoural response, 2004. http://www.ist-net.de [Online].

[5] Parke, J., Gwynne, S., Galea, E.R., and Lawrence, P. Validating the building EXODUS Evacuation Model using Data from an Unannounced Trial Evacuation. In E.R. Galea, Editor, Proceedings of 2nd International Pedestrian and Evacuation Dynamics Conference, CMS Press, Greenwich, UK (2003) ISBN 1904521088, pp. 295-306.

[6] Fraser-Mitchell, J. Simulated Evacuations of an Airport Terminal Building, Using the CRISP Model. In 2nd International Symposium in Human Behaviour in Fire (pp. 89-100). Boston, MA, 2001.

[7] Ketchell, N., Cole, S. S., and Webber, D.M. The EGRESS Code for Human Movement and Behaviour in Emergency Evacuation. In R.A. Smith and J.F. Dickie (Eds.), Engineering for Crowd Safety (pp. 361-370). London: Elsevier, 1994.

[8] Fahy, R.F. EXIT89 - An Evacuation Model for High-rise Buildings Model Description and Example Applications. In Fire Safety Science Proceedings of the 4th International Symposium (pp. 657-668), 1994.

[9] Cappuccio, J. Pathfinder: A Computer-Based Timed Egress Simulation. Fire Protection Engineering, 8, 11-12, 2000.

[10] Klupfel, H. and Meyer-König, T. Characteristics of the PedGo Software for Crowd Movement and Egress Simulation. In E.R. Galea, Editor, Proceedings of 2nd international pedestrian and evacuation dynamics conference, CMS Press, Greenwich, UK ISBN 1904521088 (pp. 331-340), 2003.

[11] Di Gangi, M., Russo, F. and Vitetta, A., A mesoscopic method for evacuation simulation on passenger ships: models and algorithms. In Pedestrian and Evacuation Dynamics 2003, pp. 197-208 CMS Press, London ISBN 1-904521-08-8, 2003.

[12] Oven, V.A., Cakici, N. Modelling the evacuation of a high-rise office building in Istanbul. Fire Safety Journal No. 44, 2009, pp. 1-15.

[13] Proulx, G., Evacuation time and movement in apartment buildings, Fire Safety Journal Volume 24, Issue 3, 1995, pp. 229-246. 
[14] Smith, R.A., Density, velocity and flow relationships for closely packed crowds, Safety Science Volume 18, Issue 4, 1995, pp. 321-327.

[15] Fang, Z., Lob, S.M., Luc, J.A. On the relationship between crowd density and movement velocity. Fire Safety Journal Volume 38, Issue 3, 2003, pp. 271-283.

[16] Kholshevnikov, V.V., Shields T.J., Boyce, K.E., Samoshin, D.A. Recent developments in pedestrian flow theory and research in Russia Fire Safety Journal No. 43, 2008, pp. 108-118.

[17] Hughes, R.L., A continuum theory for the flow of pedestrians, Transportation Research Part B, Elsevier, No. 36, 2002, pp. 507-535.

[18] Huang, L., Wong, S.C., Zhang, M., Shu, C., Lam, W.H.K. Revisiting Hughes' dynamic continuum model for pedestrian flow and the development of an efficient solution algorithm, Transportation Research Part B, Elsevier, No. 43(1), 2009, pp. 127-141.

[19] Di Gangi M., Evaluation of reliable path in risk areas, in Risk Analysis V. Southampton, U.K.: WIT Press, 2006, pp. 371-377.

[20] Di Gangi M., Musolino G., Rindone C., Vitetta A., An Italian project for exposure reduction in an urban area: experimentation design and DSS development. Proceedings of European Transport Conference. Strasbourg, Sept. 2006, ISBN/ISSN: 1-905701-01-2, 2006.

[21] Di Gangi, M., Approaching the analysis of transport networks in emergency conditions for the design of evacuation plans. In Risk Analysis IV,2004, pp. 485-494, WIT Press. ISBN 1-85312-736-1.

[22] Di Gangi M., Planning Evacuation by Means of a Multi-modal Mesoscopic Dynamic Traffic Simulation Model. B. Murgante, G. Borruso, A. Lapucci (Eds.): Geocomputation and Urban Planning, SCI 176, 2009, pp. 99-115. Springer-Verlag Berlin Heidelberg ISBN: 978-3-540-89929-7.

[23] Di Gangi M., Velonà P., Multimodal mesoscopic approach in modelling pedestrian evacuation Transportation Research Record, vol. 2090, 2009; p. 51-58, ISSN: 0361-1981, doi: 10.3141/2090-06.

[24] Di Gangi, M., Modeling Evacuation of a Transport System: Application of a Multimodal Mesoscopic Dynamic Traffic Assignment Model Intelligent Transportation Systems, IEEE Transactions on Intel. Transportation Syst., vol. 12, Issue 4, 2011, pp. 1157-1166 doi: 10.1109/TITS.2011.2143408. 